\title{
An Evaluation of Two Position based Hybrid Routing Algorithms under Mobility Speed over MANET
}

\author{
J Sandeep \\ Research Scholar \\ Department of Information \\ Technology \\ Bharathiar University \\ Coimbatore - 641046
}

\author{
T Ramesh \\ Assistant Professor \\ Department of Information \\ Technology \\ Bharathiar University \\ Coimbatore - 641046
}

\begin{abstract}
Mobile Ad Hoc Network (MANET) is collection of wireless nodes forming temporary network without any infrastructure, Ad-hoc networking is a computer communications concept. Here each node participating in the network acts as host and a router and therefore must forward packets for other nodes. Researches in this area are mostly simulation based, and in this paper we will be analyzing the performance of DWI-PBHRA routing protocol with the PBHRA. In the performance evaluation of the protocol, the protocols are tested under the realistic conditions including evaluating performance when applied to variable pause times and constant number of nodes we perform extensive simulations using NS-2 simulator.
\end{abstract}

\section{Keywords}

MANET, Position Based Routing, Ad-Hoc Networks, Mobility, Hybrid routing.

\section{INTRODUCTION}

In recent years, the study of mobile ad hoc networks (MANETs) has attracted a lot of interest, mainly from the networking community. A significant part of the research has focused on routing, which is particularly challenging in MANETs due to their dynamic nature [8], and requires algorithms that work in a fully distributed way, are able to self-organize, and show robust and adaptive behaviour. As a result, a number of MANET routing protocols have been designed [7], [3]. However, due to the costs and technological difficulty of setting up real and large MANET test beds, most of this research is carried out in simulation. These simulations are usually based on simplified scenarios, where nodes move randomly in an open area, and rely on idealized models of physical phenomena such as interference. Recently, experiences with real world test beds [2] have lead to an awareness that results from such simplified simulation scenarios do not reflect well the performance that can be expected in reality. There is therefore now a lot of interest in simulation studies that reflect more complex, realistic situations. In this paper, we investigate the distinctive properties in terms of limitations such as the mobility patterns, and data patterns, and we study how they affect the effectiveness of the two routing algorithms.

The remainder of the paper is structured as follows: Section II provides a brief overview of the related work done. Section III provides the summary of both the models, the position based hybrid routing algorithm, and the distributed workload implied position based hybrid routing algorithm. Section IV is for the design and experimental setup made for the simulation and analysis of the models. Section V will discuss the results of the simulation and analysis of the results. Finally the performance summary and the conclusions made from the results of simulation are discussed in the respective VI and VII sections.

\section{RELATED WORKS}

Extensive research has been done in modeling mobility for MANETs. In this section, we mainly focus on experimental research in this area. Much of the initial research was based on using random waypoint as the underlying mobility model and Constant Bit Rate (CBR) [5] traffic consisting of randomly chosen source-destination pairs as the traffic pattern. Routing protocols were mainly evaluated based on the following metrics: packet delivery ratio (ratio of the number of packets received to the number of packets sent) and routing overhead (number of routing control packets sent).

However, in this paper we focus on the impact of mobility models on the performance of MANET routing protocols, so our two observations regarding to discuss the effect of movement mobility speed of the nodes to evaluate the performance of the two Geographic Position Based Routing Algorithms, using NS$2[11]$ simulator considering the problem from a different perspective, using the simulation with varying number of movement speed at an invariable pause time which should be zero under weakest case because a longer pause time of the node may be insignificant for mobile Ad-hoc network with frequently and fastly moving nodes, based on the routing load and the connectivity of three typical routing protocols of ad-hoc networks with the different simulation model and metrics like (mobility speed, simulation times, connectivity sources).

\subsection{Routing Protocols for Ad-hoc Networks}

To compare and analyze mobile ad-hoc network routing protocols, appropriate classification methods are important. Classification methods as Figure 1 help researchers and workers on mobile wireless ad-hoc protocols and designers to understand distinct characteristics of a routing protocol and find its relationship with others [13]. 


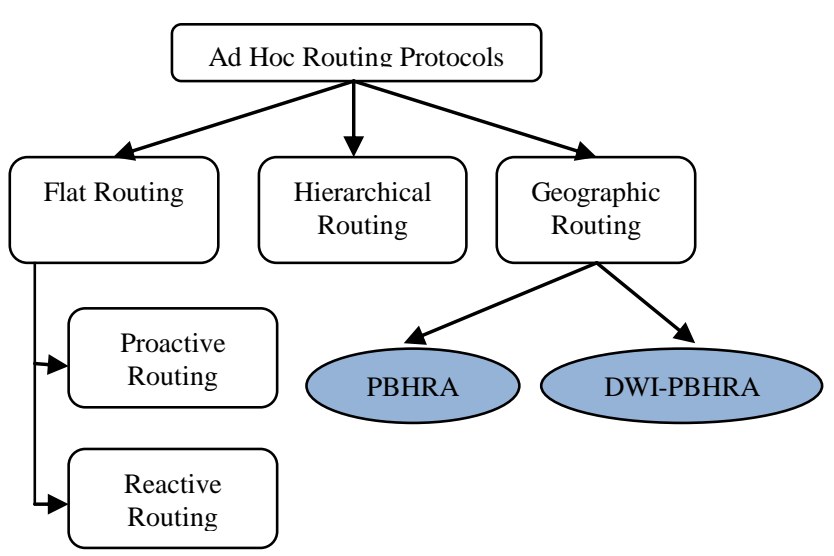

Fig 1: Classification of the Routing Algorithm

\subsection{Mobile Ad-Hoc Networks MANETs}

In order to facilitate communication within the network, a routing protocol is used to discover routes between nodes. The primary goal of such an ad-hoc network routing protocol is correct and efficient route establishment between a pair of nodes so that messages may be delivered in a timely manner. Route construction should be done with a minimum of overhead and bandwidth consumption. An Ad-hoc routing protocol is a convention or standard that controls how nodes come to agree which way to route packets between computing devices in a MANET. In ad hoc networks, nodes do not have a priori knowledge of topology of network around them, they have to discover it. The basic idea is that a new node announces its presence and listens to broadcast announcements from its neighbors. The node learns about new near nodes and ways to reach them, and announces that it can also reach those nodes. As time goes on, each node knows about all other nodes and one or more ways how to reach them.

\section{SUMMARY OF ROUTING MODELS FOR SIMULATION}

In mobile Ad hoc Network the routing can be categorized into three categories namely, proactive routing, reactive routing and hybrid routing. Many proactive protocols stem from conventional link state routing. On-demand routing, on the other hand, is a new emerging routing philosophy in the ad hoc area. It differs from conventional routing protocols in that no routing activities and no permanent routing information is maintained at network nodes if there is no communication, thus providing a scalable routing solution to large populations. His category of protocols combines the best features of the proactive and the reactive categories. Nodes within a certain distance from the node concerned, or within a particular geographical region, are said to be within the routing zone of the given node. For routing within the zone the proactive approach is used and for the nodes that are located beyond the zone a reactive approach is used. Here we will discuss the two models which will use some of the table driven or proactive and on- demand reactive routing concepts.

Although these two models comes under Hybrid protocols they differ from the other hybrid protocols as this two comes under the Geographical position based routing algorithms which are interested in localized nodes. Localization is realized by GPS that is use to determine the geographical position of the nodes. The position change occurs due to nodes mobility.

\subsection{The PBHRA Model}

Routing algorithm called position based hybrid routing algorithm (PBHRA) [9] was developed to optimize bandwidth usage of ad hoc networks. In the PBHRA algorithm, a central node, in other words a master node is assigned as it is in infrastructured wireless networks and directs the routing information. When nodes require sending data to a target node, they take the location of target node and the route to achieve it from master node. Accordingly, they send their data through that route. At this stage, the PBHRA differs from infrastructured wireless networks since data is sent via central station in infrastructured wireless networks. However in this algorithm, the master node behaving as if it is central node helps only while finding the route to achieve the target. The main goal of PBHRA is effective use of bandwidth by reducing the routing overload. Additionally, the other goals of the algorithm are to extend battery life of the mobile devices by reducing the required number of operations for route determination and to reduce the amount of memory used.

\subsection{The DWI-PBHRA Model}

The distributed workload implied approach to the PBHRA is the work with the goal of monitoring the mobility in the network and the other goals where to reduce the energy consumption for communication, reduce the routing overhead and making the network more reliable. The central nodes, in other words a primary (Brain) and secondary (Heart) nodes are assigned as it is in infrastructured wireless networks and directs the routing information. When nodes require sending data to a target node, they take the location of target node and the route to achieve it from brain node. Accordingly, the data is send through that route. The heart node is newly implemented to monitor the networks mobility by broadcasting frequent alive packets in the network. And the algorithm provides the network with the recovery mechanism, for unexpected crashes of Master, making the network more reliable.

\section{DESIGN OF THE EXPERIMENT \& SIMULATION SETUP}

The Algorithms to be analyzed for routing need to be experimented under the carefully designed routing traffic base configuration and network scenario, and to vary the node density and mobility at a time to stress the network in different directions. Careful selection of these control parameters enables us to assess and isolate the effect of network size, with fixed application traffic CBR. In addition, design of the base condition, network topology, and routing are to be taken into account the real networks for which the results should be applicable.

In this experiment, we noted down the energy loss, routing overhead, and Packet delivery fraction for various mobility pause times and a multimode scenarios for assessing the scalability issue for the routing protocols under consideration. In the beginning of the experiment, the initial settings of the node 
and simulation times were thoroughly checked out. Care also is taken in selection of the terrain dimension, disabling the unnecessary filter components in the simulator settings. The experiment is continued for different node pause time of 20,40 , 60, 80 and 100 seconds respectively. In all these cases, we noted down the energy loss, routing overhead, and Packet delivery fraction in the simulator. We selected the terrain dimensions as $500 \mathrm{~m} \times 500 \mathrm{~m}$, and nodes in the terrain are mobile.

We fixed the simulation time, initial energy, network density and other settings of the simulator, and firstly with the pause time 20 and then with varied values from 20 to 100 seconds the experiment is made in the network. The NS2 used to simulate the ad-hoc routing protocols in is the Network Simulator 2 (ns) from Berkeley. Due to its flexibility and modular nature, it is a discrete event simulator targeted at networking research. It provides substantial support for simulation of TCP, routing, and multicast protocols. The simulator is written in $\mathrm{C}++$ and a script language called OTcl.

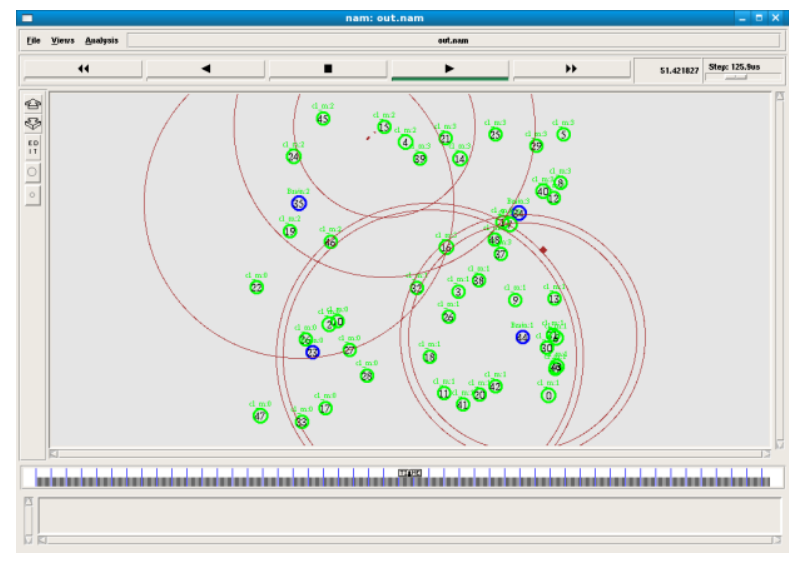

Fig 2: Screen Shot of PBHRA

The Figure 2 is the nam output of the Position Based Hybrid Routing Algorithm, this simulation is made where the 50 nodes have been spread randomly in the area of $500 \times 500 \mathrm{~m}$, and the algorithm starts at the time of simulation time $40 \mathrm{sec}$ where the algorithm clusters the nodes into 4 clusters and with relative cluster heads, for which it uses an gird clustering algorithm, the blue node in the figure shows the cluster head and the green nodes are the member nodes once the clusters and there cluster heads are formed the next phase of the algorithm finds the routing path for every node to other by the cluster head. This routing path are requested by the source nodes for the transfer of data to destination, in response to the request the cluster head sends the path of destination to the source node. The source sends the packets through the route send by the cluster head. Here the shortest paths are calculated and the updating of path all done by the single cluster head.

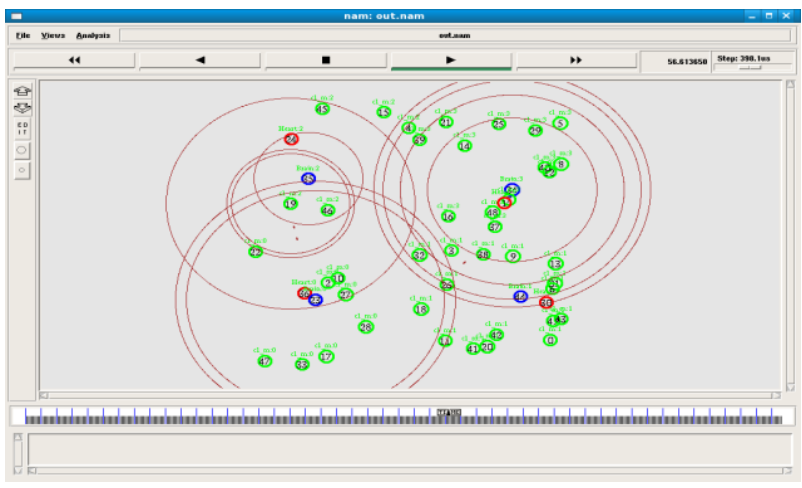

Fig 3: Screen Shot of DWI-PBHRA

The Figure 3 is the nam output of the Distributed workload implied Position Based Hybrid Routing Algorithm, this simulation is made where the 50 nodes have been spread randomly in the area of $500 \times 500 \mathrm{~m}$, and the algorithm starts at the time of simulation time 40sec. at the first phase of the algorithm the nodes are clustered by the gird clustering algorithm, and the clustering algorithm also finds the central nodes for the candidature of brain and Heart of the cluster. The brain (primary masters) node which maintains the table of routing path and responds the requests of source nodes for the path to destination. The heart (secondary master) node of the cluster monitors the mobility of the network. The remaining nodes are the member nodes of the network.

\subsection{Mobility Setup}

A mobility model [10] should attempt to mimic the movements of real Mobile Networks. Changes in speed and direction must occur and they must occur in reasonable time slots. For example, we would not want Mobile Networks to travel in straight lines at constant speeds throughout the course of the entire simulation because real Mobile Networks would not travel in such a restricted manner. There is several mobility models supported, nodes in the simulation set up move according to a model that is well known as the "random waypoint" model. The movement scenario files we used for each simulation are characterized by a pause time. Each node begins the simulation by remaining stationary for pause time seconds. It then selects a random destination in the $500 \mathrm{~m} \times 500 \mathrm{~m}$ space and moves to that destination at a speed distributed uniformly between $0 \mathrm{mps}$ and a maximum speed of 10mps. Upon reaching the destination, the node pauses again for pause time seconds, selects another destination, and proceeds there as previously described, repeating this behavior for the duration of the simulation. Each simulation ran for 200 seconds of simulated time. We ran our simulations with movement patterns generated for a fixed pause time of 30 Seconds.

\subsection{Traffic Setup}

A traffic generator named Cbrgen was developed to simulate constant bit rate sources in NS-2, act as the important parameter of our simulation to compare the performance of each routing protocol. We chose our application traffic sources to be constant bit rate (CBR) sources. When defining the parameters of the communication model, we experimented with sending rates of 1.2 packets per second and packet sizes of 512 bytes to observe the consistency. 


\subsection{Effect of Unvarying Pause Time}

Pause time can be defined as time for which nodes waits on a destination before moving to other destination. We used a constant pause time as a parameter as it is measure of mobility of nodes. Low pause time means no de will wait for less time thus giving rise to high mobility scenario.

\section{SIMULATION RESULTS AND PERFORMANCE}

This section presents a comparative analysis of the performance metrics generated from all simulations, evincing general and relevant aspects of the evaluated routing protocols in the diversity of network mobility levels that can occur over the Position Based Hybrid Routing Algorithm, and Distributed Workload implied Position Based Hybrid Routing Algorithm. Considering the diversity of routing protocols user mobility levels $(20,40,60,80$, and $100 \mathrm{~m} / \mathrm{s})$.

Performance metrics that have been proposed for the performance evaluation of an ad-hoc network protocol. The following metrics are applied to comparing the protocol performance. Some of these metrics are suggested by the MANET working group for routing protocol evaluation [6].

Packet delivery fraction ratio: The ratio between the number of data packets originated by the "application layer" CBR sources and the number of data packets received by the CBR sink at the final destination [1], [12].

Routing packet overhead: Routing Packet overhead RPO is the total number of transmissions routing packets transmitted during the simulation. For packets sent over multiple hops, each transmission of the packet (each hop) counts as one transmission [4].

Packet loss ratio: The ratio of the data packets originated by the sources failure to deliver to the destination.

\subsection{Analysis Based on Energy Consumed by Cluster-heads}

The Energy consumed by the cluster heads were compared for both the algorithms under the diversity of routing protocols user mobility levels, and the results shown in the Figure 4 suggests that in the DWI-PBHRA model the cluster heads consumed lesser energy compare to the PBHRA at any mobility levels.

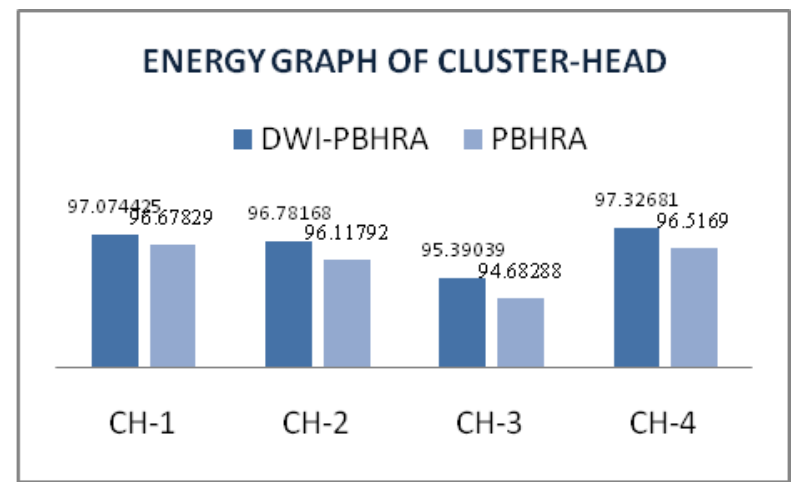

Fig 4: Energy Graph of Cluster-Head

\subsection{Analysis Based on Packet Routing Overhead}

The routing packet overhead is the packets need to be transferred to make a connection for communication, and from the results of the simulation the DWI-PBHRA performed better with less packet routing overhead in the network at the various levels of the mobility.

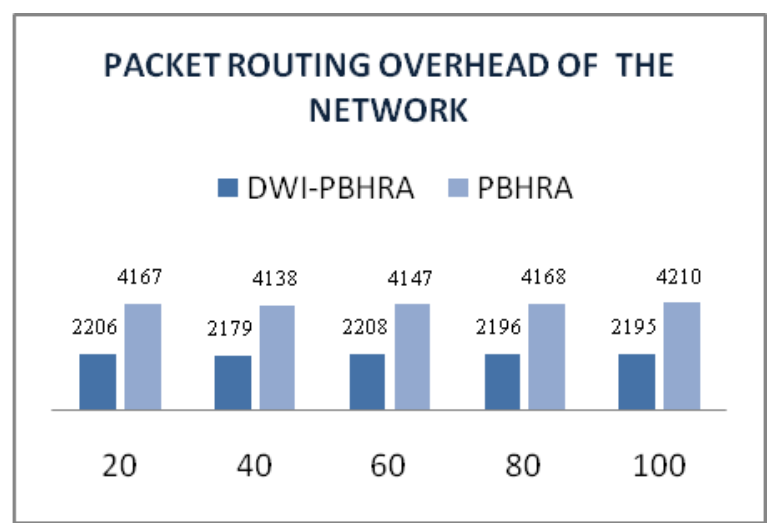

Fig 5: Packet Routing Overhead of the Network

The Figure 5 is the graph with the $\mathrm{x}$ axis for variable pause time and the $\mathrm{y}$ axis is for the no of packets for the network to establish and connection to be made for the transfer of the data in the network. This analysis was made under the fixed simulation setup where the simulation was made for 200 secs.

\subsection{Analysis Based on Packet Delivery Fraction}

This metric which we call the ratio of delivered packets is an important as it describes the loss rate that will $b$ e seen by the transport protocols, which in turn affects the maximum throughput that the network can support., the two models where compared for the packet delivery fraction and the results in the Figure 6 suggests the distributed workload implied position based hybrid routing algorithm gives a better result with higher packet delivery fractions at all the mobility levels.

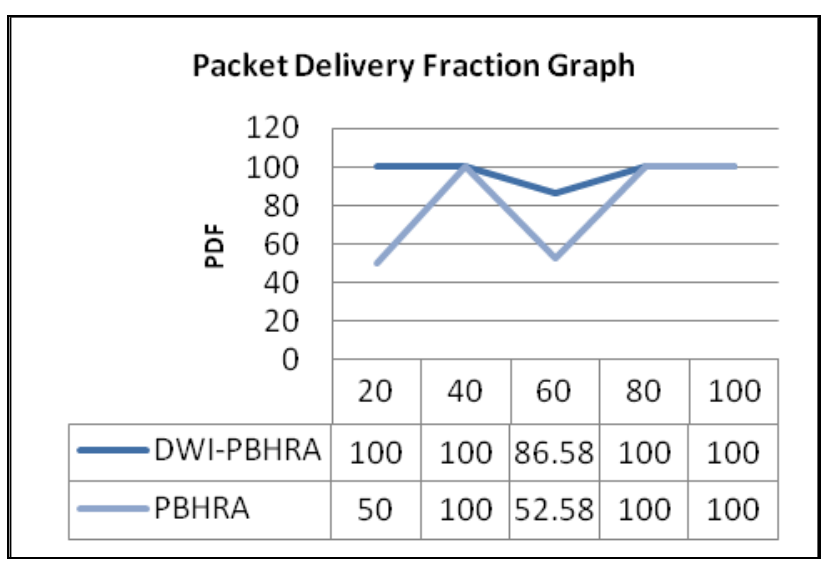

Fig 6: Packet Delivery Fraction in the Network 


\section{PERFORMANCE ANALYSIS}

Performance analysis has done for the pause time with packets received, pause time with consumed energy. During the simulation it was found that a change in the pause time affects the energy consumption and packet delivery fractions in the network. Every time the network needs to be monitored and every hop need to reliable for transfer of data hence topology changes frequently need new routes for data transfer. It was found that for more mobility or short pause time was not managed well in the infrastructure less network where the infrastructure based network with central control were more capable of handling large networks better in high mobility. Here our proposed scheme has given better results in terms of packed delivery fractions as well as energy savings.

\section{CONCLUSION}

The area of ad-hoc networking has been receiving increasing attention among researchers in recent years, as the available wireless networking and mobile computing hardware bases are now capable of supporting the promise of this technology. Over the past few years, a variety of new routing proto cols targeted specifically at the ad-ho c networking environment have been proposed, but little performance information on each protocol and no de tailed performance comparison between the proto cols has previously been available.

This paper has presented a comparing performance of two Position Based routing algorithms that comes under the hybrid routing algorithm category, include the properties of table driven and on demand protocols and are usually interested in localized nodes. For routing packets between wirelesses mobile hosts in an ad-hoc network PBHRA and DWI-PBHRA using a network simulator like NS-2 with scenario consist of fixed network size, number of nodes and movement speed in a range of 0 to 10 at variable pause time.

The General observation from the simulation:

The Distributed workload implied position based routing algorithm which was developed with the goal of monitoring the mobility of the network that it achieved by introduction of heart node and its pulses, The Performance got a difference because of the distribution of the work among the member, Brain and Heart nodes. Whereas the master node in the Position base Hybrid routing algorithm had more overload of routing and giving central control, the reliability was there in the new approach of brain and heart with its recovery mechanism from crash of Master.

The Results showed the distributed workload implied position based hybrid routing algorithm outperformed the position based hybrid routing algorithm for the energy consumption by cluster head, packet Routing overhead, and packet delivery fraction.

\section{REFERENCES}

[1] C. E. Perkins, E. M. Royer, S. R. Das, and M. K. Marina, "Performance comparison of two on-demand routing proto cols for Ad Ho c networks," IEEE Personal Communications Magazine Special Issue on Ad hoc Networking , vol. 8, pp. 16-28, Feb. 2001.

[2] C. Tschudin, P. Gunningberg, H. Lundgren, and E.
Nordstrom, "Lessons from experimental MANET research," Ad Hoc Networks Journal, vol.3, no.2, pp.221233, 2005.

[3] E.Royerand, C. K. Toh, "A review of current routing protocols for ad hoc mobile wireless networks," IEEE Personal Communications, 1999.

[4] J. Bro ch, D. Johnson, and D. Maltz, The Dynamic Source Routing Protocol for Mobile Ad Hoc Networks, IETF Internet Draft, Dec. 1998. (http://www.ietf.org/internetdrafts/draft-ietfmanet-dsr-01.txt)

[5] Kevin Fall and Kannan Varadhan, "ns notes and documentation". The VINT project, UC Berkeley, LBL, USC/ISI, and Xerox PARC, May 1998.

[6] L. Layuan, Y. Peiyan, and L. Chunlin, "Performance evaluation and simulations of routing protocols in Ad ho c networks," Computer Communications, vol. 30, pp. 18901998, 2007.

[7] M. Abolhasan, T. Wysocki, and E. Dutkiewicz, "A review of routing protocols for mobile ad hoc networks," Ad Hoc Networks, vol.2, pp. 1-22, 2004.

[8] N. H. Vaidya, "Mobile Ad ho c networks routing, mac and transport issues," Proceedings of the IEEE International Conference on Computer Communication INFOCOM, 2004.

[9] Resul Kara ,Ibrahim Ozcelik and Huseyin Ekiz (2010). Anew routing algorithm in MANETs: Position based hybrid routing. Scientific research and essays Vol. 5(3), pp. $328-238$.

[10] T. Camp, J. Boleng, and V. Davies, "A survey of mobility mo dels for Ad ho c network research," Wireless Communications \& Mobile Computing (WCMC), Special issue on Mobile Ad Hoc Networking, Research, Trends and Applications, Sep. 2002.

[11] The Network Simulator - NS-2. (http://www.isi.edu /nsnam/ns/index.html).

[12] Y. Wang, V. C. Giruka, and M. Singhal, "A truthful geographic forwarding algorithm for Ad ho c networks with selflsh nodes," Proceedings of the International Journal of Network Security, Nov. 2007.

[13] Yasser Kamal Hassan, Mohamed Hashim Ab d El-Aziz, and Ahmed Safwat Ab d El-Radi, "Performance Evaluation of Mobility Speed over MANET Routing Protocols" International Journal of Network Security, Vol.11, No.3, pp.128-138, Nov. 2010. 